\title{
Stage IB Eyelid Carcinoma AJCC v8
}

National Cancer Institute

\section{Source}

National Cancer Institute. Stage IB Eyelid Carcinoma A/CC v8. NCI Thesaurus. Code C140518.

Stage IB includes: T2a, N0, M0. T2a: Tumor measuring more than $10 \mathrm{~mm}$ but $20 \mathrm{~mm}$ or less in greatest dimension without invasion of the tarsal plate or eyelid margin. N0: No evidence of lymph node involvement. M0: No distant metastasis. (AJCC 8th ed.) 